\title{
The Schottky-Klein prime function and counting functions for Fenchel double crosses
}

\author{
M. Pollicott ${ }^{1}$
}

Received: 18 May 2020 / Accepted: 25 March 2021 / Published online: 15 April 2021

(C) The Author(s) 2021

\section{Abstract}

We relate the classical nineteenth century Schottky-Klein function in complex analysis to a counting problem for pairs of geodesics in hyperbolic geometry studied by Fenchel. We then solve the counting problem using ideas from ergodic theory and thermodynamic formalism.

Keywords Schottky-Klein function · Schottky groups · Orbital Counting Function

Mathematics Subject Classification Primary 30F40 - 37D35; Secondary 37D40 . 30E99

\section{Introduction}

A Kleinian group is a discrete group of isometries of three dimensional hyperbolic space $\mathbb{H}^{3}$ with respect to the Poincaré metric. A classical Schottky group is a special case which is isomorphic to a free group. These are particularly simple examples of Kleinian groups, for which the limit set in the Riemann sphere $\widehat{\mathbb{C}}$ is a Cantor set. It is often convenient to describe the elements of the group in terms of linear fractional transformations of $\widehat{\mathbb{C}}$.

The Schottky-Klein prime function is a function of two complex variables. In particular, it can be defined as a function of two points in the Riemann sphere, outside of the limit set. The Schottky-Klein function was extensively studied in the nineteenth

Communicated by Adrian Constantin.

I am grateful to J. Parkkonen for correcting an error in an earlier version of this note and to F. Paulin for bringing to my attention his work with Parkkonen. I would like to thank the referee for his illuminating comments and suggestions.

\footnotetext{
M. Pollicott

mpollic@maths.warwick.ac.uk

1 Mathematics Institute, University of Warwick, Coventry CV4 7AL, UK
} 
century. It came back into modern usage in the last decade in the generalization of the classical Schwarz-Christoffel theorem to multiply connected regions and myriad other applications. A comprehensive recent reference is the book [7].

\subsection{The Schottky group and the Schottky-Klein function}

We begin with a definition of the Schottky groups we will be studying. Let $D_{i}, D_{i}^{\prime}$ for $i=1, \ldots, d$, be disjoint disks in $\mathbb{C}$. Let $g_{i}: \widehat{\mathbb{C}} \rightarrow \widehat{\mathbb{C}}$ be the Möbius transform which maps $D_{i}$ onto $\widehat{\mathbb{C}}-\operatorname{int}\left(D_{i}^{\prime}\right)$. The associated Schottky group $\Gamma=\left\langle g_{1}, \ldots, g_{d}\right\rangle$ is defined to be the free group they generate.

Definition 1.1 The limit set $\Lambda=\Lambda(\Gamma)$ is the set of accumulation points of $\Gamma 0$ in $\widehat{\mathbb{C}}$.

We denote by $F=\widehat{\mathbb{C}}-\cup_{i=1}^{d}\left(D_{i} \cup D_{i}^{\prime}\right)$ the complex region in the complex plane exterior to all of the circles. We can then write $\widehat{\mathbb{C}}-\Lambda=\left(\cup_{g \in \Gamma} g F\right)$. Given a Schottky group and two distinct points in $F$ we can associate the value of the Schottky-Klein prime function.

Definition 1.2 The Schottky-Klein prime function is given by

$$
w(z, \xi):=(z-\xi) \prod_{g \in \Gamma_{0}} \frac{(g z-\xi)(g \xi-z)}{(g z-z)(g \xi-\xi)}, \quad \text { where } z, \xi \in F
$$

whenever it converges, where the product is restricted to those elements of the Schottky group generated by $g_{1}, \ldots, g_{d}$, excluding the identity and taking only an element or its inverse (but not both).

\subsection{An asymptotic estimate}

A closely related question is to understand the individual terms in the product. Heuristically, convergence of the infinite product means that the individual terms converge to 1 quickly enough. In fact, we shall show values converge to 1 in a uniform way.

Let $0<\delta<2$ denote the Hausdorff dimension $\operatorname{dim}_{H}(\Lambda)$ of $\Lambda$.

Theorem 1.3 There exists $C>0$ such that

$$
\text { Card }\left\{g \in \Gamma:\left|\frac{(g z-\xi)(g \xi-z)}{(g z-z)(g \xi-\xi)}-1\right| \geq \frac{1}{T}\right\} \sim C T^{\delta}
$$

as $T \rightarrow+\infty$. 2.

Replacing $\Gamma$ by $\Gamma_{0}$ in (1.2) gives a similar asymptotic, differing only by a factor of

As usual, the asymptotic (1.2) means that the ratio of the Left Hand Side of (1.2) to the Right Hand Side converges to unity as $T \rightarrow+\infty$. By a result of Doyle there exists a uniform bound $\operatorname{dim}_{H}(\Lambda) \leq \beta<2[10]$. 
The proof of Theorem 1.3 will come by reformulating it in terms of a rather natural geometric counting problem for Fenchel double crosses. We refer the reader to Sect. 3 for more details.

\subsection{The Fenchel double cross}

The above asymptotic (1.2) is equivalent to a natural geometric result. Let $\mathbb{H}^{3}$ be three dimensional hyperbolic space, with the hyperbolic metric $d$, and let $\Gamma$ be a Schottky group. Let $\gamma$ be a geodesic in $\mathbb{H}^{3}$ whose end points $\gamma(-\infty), \gamma(+\infty)$ are disjoint from the limit set $\Lambda$. The analogue of (1.2) is the following.

Theorem 1.4 There exists $C>0$ such that

$$
\text { Card }\{g \in \Gamma: d(\gamma, g \gamma) \leq T\} \sim C e^{\delta T}
$$

as $T \rightarrow+\infty$.

We have formulated this as a counting function for a Schottky group, so as to correspond to the counting function for the cross-ratio. However, it is easy to adapt the proof to the case a more general convex co-compact Kleinian group. Parkkonen and Paulin have announced more general results [18], but for the present setting our method appears more succinct.

\section{Convergence of the Schottky-Klein prime function}

The Schottky-Klein prime function was a object of considerable interest in the nineteenth century and was studied, for example, by Schottky in his 1887 article [25] and by Klein in his 1890 article [16]. Interest was recently revived due to its application to generalizations of the classical Chrstoffel-Schwarz theorem [4-6,9].

A basic problem to address is whether the product defining $w(z, \xi)$ actually converges. This particular problem was discussed by Baker in his 1897 book [1,3]. The classical approach to convergence was to use a hypothesis called "circle decomposible" (cf. [2, p. 58]). Heuristically, we see that there is convergence if the circles are not too close together (which also leads to a sufficient condition for $\operatorname{dim}_{H}(\Lambda)$ to be small). An interesting discussion of this general problem is given in [6].

It is convenient to formulate this in terms of the Hausdorff dimension $\delta$ of the limit set $\Lambda$. The following result is a simple corollary of Theorem 1.3.

Corollary 2.1 If the terms in the product (1.1) are ordered by the distance of their absolute values from 1 then the Schottky-Klein function converges provided the dimension $\delta$ of the limit set is strictly smaller than 1.

We can consider a simple example.

Example 2.2 (A doubly connected domain) We can consider the circles

$$
C_{1}^{ \pm}=\left\{z \in \mathbb{C}:\left|z \pm \frac{3}{2}\right|=\frac{1}{2}\right\} \text { and } C_{2}^{ \pm}=\left\{z \in \mathbb{C}:\left|z \pm \frac{5}{12}\right|=\frac{1}{12}\right\} .
$$




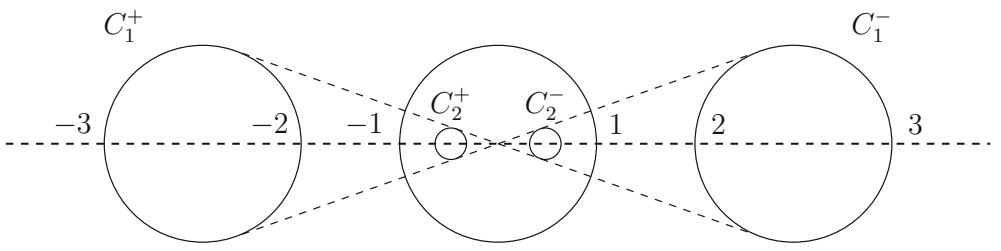

Fig. 1 A specific example of a Kleinian group

We can choose linear fractional transformations

$$
g_{1}(z)=\frac{5-2 z}{12-5 z} \text { and } g_{2}(z)=\frac{-5-2 z}{12+5 z}
$$

such that $g_{1}\left(C_{1}^{-}\right)=C_{2}^{-}$by $g_{2}\left(C_{1}^{+}\right)=C_{2}^{+}$corresponding to generators of a Schottky group $\Gamma=\left\langle g_{1}, g_{2}\right\rangle$ (Fig. 1). In this case the Hausdorff dimension has an approximate value $\operatorname{dim}_{H}(\Lambda)=0.29 \ldots$ Since the set $\Lambda$ is the limit set of a Schottky Kleinian group it can characterized in terms of the pressure function and dynamical zeta functions in ergodic theory. This approach was used to numerically estimate $\operatorname{dim}_{H}(\Lambda)$ and we refer the reader to [15] for more details.

Remark 2.3 This theorem is also suggested by the close connection with Poincaré series

$$
\mathcal{P}(s)=\sum_{g \in \Gamma}\left|g^{\prime}(0)\right|^{-s}
$$

(see also the appendix to [8]). It follows from work of Ruelle that $\mathcal{P}(s)$ converges provided $\operatorname{Re}(s)>\delta=\operatorname{dim}_{H}(\Lambda)$, the Hausdorff of the limit set [23].

In the case that the series doesn't converge the function can still be defined in terms of an analytic function with appropriate transformation properties (cf. [14]). Even in the case of convergence there is the practical issue of the speed of approximation (cf. Sect. 7).

Remark 2.4 There are a simple class of Schottky groups which play an important role in extending the Schwarz-Christoffel theorem to multiply connected domains [6]. Let $z_{1}, \ldots, z_{d} \in \mathbb{D}=\{z \in \mathbb{C}:|z|<1\}$ be the centres of subdisks

$$
\mathbb{D}_{i}=B\left(z_{i}, r_{i}\right)=\left\{z \in \mathbb{D}:\left|z-z_{i}\right|<r_{i}\right\}
$$

where $\left|z_{i}\right|+r_{i}<1$.

We can then associate a "multiply connected region" defined by $\mathbb{M}=\mathbb{D}-\cup_{i=1}^{d} \mathbb{D}_{i}$. When $d=1$ this simply corresponds to an annulus. Similarly, we can consider subpolygons $\mathbb{P}_{1}, \ldots, \mathbb{P}_{d} \subset \mathbb{P}$ with sets of vertices $V_{1}, \ldots, V_{d}$, respectively. We then let $\mathbb{Q}=\mathbb{P}-\cup_{i=1}^{d} \mathbb{P}_{i}$ 


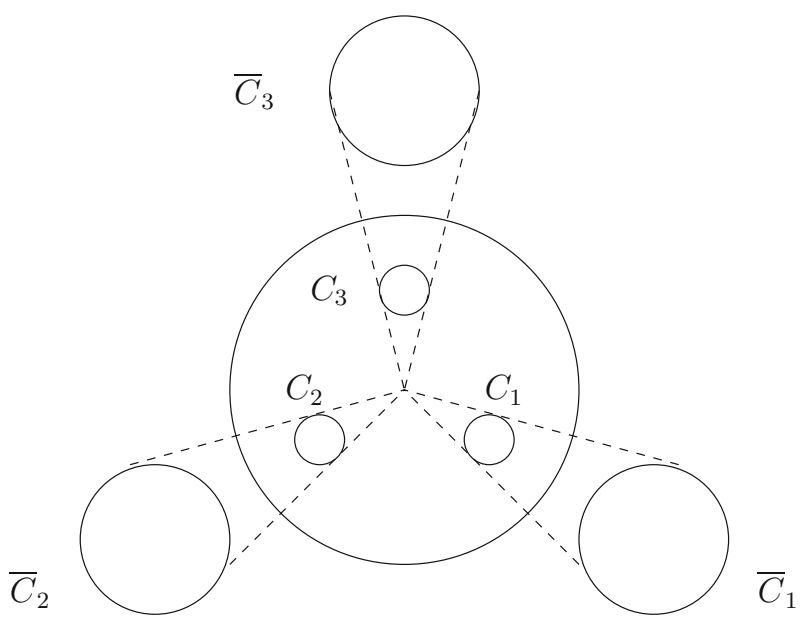

Fig. 2 A particular example of a Kleinian group relevant to generalizations of the Schwarz-Christoffel theorem. One classical example involves choosing disjoint disks $\mathbb{D}_{1}, \mathbb{D}_{2}, \mathbb{D}_{3}$ with boundaries $C_{i}=\partial \mathbb{D}$ and their images $\bar{C}_{i}(i=1,2,3)$ corresponding to reflections in the unit circle

Let $C_{i}=\partial \mathbb{D}_{i}$ and let $\bar{C}_{i}$ denote the images on reflection in the unit circle $\{z \in$ $\mathbb{C}:|z|=1\}=\partial \mathbb{D}$ (Fig. 2). In particular, $g_{i}(z)=z_{i}+\frac{r_{i}^{2} z}{1-z_{i} z}$ satisfies $g_{i}\left(\bar{C}_{i}\right)=C_{i}$. Let $\Gamma=\left\langle g_{1}, \ldots, g_{d}\right\rangle$ be the associated Schottky group. By a result of Crowdy [4,5] there is an explicit formula for a holomorphic bijection $\psi: \mathbb{M} \rightarrow \mathbb{Q}$ given by

$$
\psi(z)=\int_{z_{0}}^{z} C(\cdot) \prod_{i=1}^{k} w\left(z, w_{i}\right)^{\alpha_{i}} \prod_{i=1}^{d} \prod_{j=1}^{\left|V_{i}\right|} w\left(z, w_{j}^{(i)}\right)^{\alpha_{i}^{(j)}} d z
$$

where $w(\cdot, \cdot)$ is the Schottky-Klein prime function and

(1) the exponents $\alpha_{1}, \ldots, \alpha_{k}$ correspond to the internal angles of $\mathbb{P}$ and exponents $\alpha_{1}^{(j)}, \ldots, \alpha_{n_{\left|V_{i}\right|}}^{(j)}$ correspond to the internal angles of $\mathbb{P}^{(j)}$

(2) the points $W=\left\{w_{1}, \ldots, w_{k}\right\} \in \partial D$ on the unit circle correspond to preimages of the vertices $V$ and the points $W^{(i)}=\left\{w_{1}^{(i)}, \ldots, w_{\left|V_{i}\right|}^{(i)}\right\} \in \partial D_{i}$ on the circle correspond to preimages of the vertices $V_{i}$;

(3) $C(\cdot)$ is an explicit correction function.

\section{Hyperbolic geometry and double crosses}

In this section we want to describe the action of the Schottky group on three dimensional hyperbolic space. Let $\Gamma=\left\langle g_{1}, \ldots, g_{d}\right\rangle$ be a Schottky group with generators $g_{1}, \ldots, g_{d}$. We let

$$
\mathbb{H}^{3}=\{(z, t): z=x+i y \in \mathbb{C}, t>0\}
$$


denote the upper half-space with the Poincaré metric

$$
d s^{2}=\frac{d x^{2}+d y^{2}+d t^{2}}{t^{2}}
$$

The group $\Gamma$ acts on $\mathbb{H}^{3}$ via linear fractional transformations on the boundary, i.e., if $g \in \Gamma$ we have that

$$
g(z)=\frac{a z+b}{c z+d} \quad \text { where } g=\left(\begin{array}{ll}
a & b \\
c & d
\end{array}\right) .
$$

We are particularly interested in counting problems. We begin by recalling the classical counting function for the orbits of the point $j=(0,1) \in \mathbb{H}^{3}$.

Definition 3.1 We denote by

$$
N_{0}(T)=\operatorname{Card}\{g \in \Gamma: d(g j, j) \leq T\}
$$

for $T>0$.

Counting functions of this general type have been extensively studied cf. [20-22]. We recall the appropriate asymptotic formulae for this function [21].

Theorem 3.2 (Hyperbolic circle problem) There exists $C>0$ such that

$$
N_{0}(T) \sim C e^{\delta T} \text { as } T \rightarrow+\infty
$$

i.e. $\lim _{T \rightarrow+\infty} N_{0}(T) e^{-\delta T}=C$.

We want to consider an analogous problem where we replace the $\Gamma$-orbit of the point $j$ by the $\Gamma$-orbit of a geodesic $\gamma$ in $\mathbb{H}^{3}$. The image $g=g(\gamma)$ is again a geodesic. We denote the usual distance between $\gamma$ and $g \gamma$ by

$$
d(\gamma, g \gamma)=\inf _{t_{1}, t_{2} \in \mathbb{R}} d\left(\gamma\left(t_{1}\right), g \gamma\left(t_{2}\right)\right)
$$

where $\gamma: \mathbb{R} \rightarrow \mathbb{H}^{3}$ and $g \gamma: \mathbb{R} \rightarrow \mathbb{H}^{3}$ denote the parameterizations of the geodesics $\gamma$ and its image $g \gamma$. Let us denote by $\tau$ the shortest geodesic between $\gamma$ and $g \gamma$.

Following Fenchel, we can describe the configuration of the three geodesics $\gamma, g \gamma$ and $\tau$ as a double cross. (This nomenclature apparently refers to the geodesics $\gamma$ and $g \gamma$ both crossing at third geodesic, part of which is denoted as a dashed curve in Fig. 3.)

Definition 3.3 We denote by

$$
N(T)=\operatorname{Card}\{g \in \Gamma: d(\gamma, g \gamma) \leq T\}
$$

for $T>0$. 


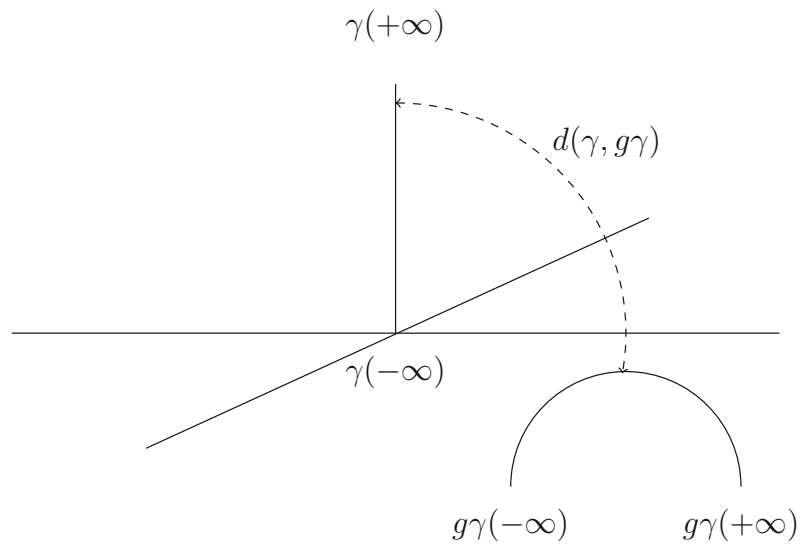

Fig. 3 The Fenchel double cross: the distance $d(\gamma, g \gamma)$ between $\gamma$ and $g \gamma$

We now have the following analogue of the previous theorem (which is merely a restatement of Theorem 1.3).

Theorem 3.4 There exists $C>0$ such that $N(T) \sim C e^{\delta T}$ as $T \rightarrow+\infty$.

We will prove this in the next two sections.

\section{Complex cross ratios}

We recall the definition of a complex cross ratio for four distinct points in the Riemann sphere $\widehat{\mathbb{C}}$.

Definition 4.1 We define the cross ratio by

$$
R\left(z_{1}, z_{2}, z_{3}, z_{4}\right)=\frac{\left(z_{1}-z_{3}\right)\left(z_{2}-z_{4}\right)}{\left(z_{2}-z_{3}\right)\left(z_{1}-z_{4}\right)}
$$

for distinct $z_{1}, z_{2}, z_{3}, z_{4} \in \widehat{\mathbb{C}}$.

Following Fenchel and Ahlfors we can consider a geodesic $\gamma$ in $\mathbb{H}^{3}$ with endpoints $z_{1}=\gamma(-\infty)$ and $z_{2}=\gamma(+\infty)$. Given $g \in \Gamma$ we can consider the image geodesic $g \gamma$ with endpoints $z_{3}=(g \gamma)(-\infty)$ and $z_{4}=(g \gamma)(+\infty)$. The complex distance $\mu=\mu(g) \in \mathbb{C}$ from $\gamma$ to $g \gamma$ is such that the modulus $|\mu|=d(g \gamma, \gamma)$ is the length of their common normal and $\mu /|\mu|=e^{i \theta}$, where $\theta$ is the relative angle between $\gamma$ and $g \gamma$ along their normal (i.e., the change in the angle by parallel transporting a frame along the geodesic realizing this distance).

The connection between the cross ratio and the geometry is the following.

Lemma 4.2 [12, p. 68, equation (3)]

$$
R\left(z_{1}, z_{2}, z_{3}, z_{4}\right)=\tanh ^{2}(\mu / 2)=\left(\frac{e^{\mu}-1}{e^{\mu}+1}\right)^{2}
$$


Proof of Theorem 1.3 assuming Theorem 1.4 Given $z, \xi \in F$, we let $\gamma$ be a geodesic in $\mathbb{H}^{3}$ with these endpoints. Using Lemma 4.2 we can write

$$
\begin{aligned}
\left|\frac{(g z-\xi)(g \xi-z)}{(g z-z)(g \xi-\xi)}-1\right| & =\left|\left(\frac{e^{\mu}-1}{e^{\mu}+1}\right)^{2}-1\right| \\
& =\frac{4\left|e^{\mu}\right|}{\left|e^{\mu}+1\right|^{2}} \sim 4 e^{-d(\gamma, g \gamma)}
\end{aligned}
$$

as $d(\gamma, g \gamma) \rightarrow+\infty$. Moreover, by Theorem 1.4 we have that

$$
\operatorname{Card}\{g \in \Gamma: d(\gamma, g \gamma) \leq T\} \sim C T^{\delta},
$$

as $T \rightarrow+\infty$. Finally, comparing (4.1) and (4.2) completes the proof of Theorem 1.3.

\section{Symbolic dynamics and transfer operators for Hölder functions}

The method of proof of the asymptotic formulae in the previous sections uses ideas from symbolic dynamics and thermodynamic formalism. In particular, we can associate to the counting problem an analytic function analogous to the Poincare series.

\subsection{The subshift of finite type}

Following a standard approach, we can code the limit set $\Lambda$ by infinite sequences. It is convenient to label the generators (and their inverses) for $\Lambda$ by $g_{1}, \ldots, g_{d}, g_{d+1}, \ldots, g_{2 d}$ with $g_{j+n}=g_{j}^{-1}$, for $1 \leq j \leq n$. In particular, the points in the limit set can be coded by infinite sequences with the $2 d \times 2 d$ transition matrix $A$, with rows and columns indexed by the generators and their inverses, defined by

$$
A=\left(\begin{array}{cccccccc}
1 & 1 & 1 & \cdots & 0 & 1 & 1 & \ldots \\
1 & 1 & 1 & \cdots & 1 & 0 & 1 & \ldots \\
1 & 1 & 1 & \cdots & 1 & 1 & 0 & \ldots \\
\vdots & \vdots & \vdots & \vdots & \vdots & \vdots & \vdots & \vdots \\
0 & 1 & 1 & \cdots & 1 & 1 & 1 & \ldots \\
1 & 0 & 1 & \cdots & 1 & 1 & 1 & \cdots \\
1 & 1 & 0 & \cdots & 1 & 1 & 1 & \cdots \\
\vdots & \vdots & \vdots & \ldots & \vdots & \vdots & \vdots & \vdots
\end{array}\right),
$$

i.e.,

$$
A(i, j)= \begin{cases}1 & \text { if }|i-j| \neq d \\ 0 & \text { if }|i-j|=d .\end{cases}
$$


We can denote

$$
\Sigma=\left\{\left(i_{n}\right)_{n=0}^{\infty} \in \prod_{n=0}^{\infty}\{1,2, \ldots, 2 d\}: A\left(i_{n}, i_{n+1}\right)=1 \text { for } n \geq 0\right\}
$$

The space $\Sigma$ is compact with respect the metric

$$
d\left(\left(i_{n}\right)_{n=0}^{\infty},\left(j_{n}\right)_{n=0}^{\infty}\right)=\sum_{n=0}^{\infty} \frac{e\left(i_{n}, j_{n}\right)}{2^{n}} \quad \text { where } e(i, j)= \begin{cases}1 & \text { if } i \neq j \\ 0 & \text { if } i=j\end{cases}
$$

Let $\sigma: \Sigma \rightarrow \Sigma$ denote the usual continuous shift map given by $\sigma\left(i_{n}\right)_{n=0}^{\infty}=\left(i_{n+1}\right)_{n=0}^{\infty}$.

Example 5.1 When $d=2$ we can consider generators $g_{1}, g_{2}$ and their inverses $g_{1}^{-1}$, $g_{2}^{-1}$. The associated matrix takes the form

$$
A=\left(\begin{array}{llll}
1 & 0 & 1 & 1 \\
0 & 1 & 1 & 1 \\
1 & 1 & 1 & 0 \\
1 & 1 & 0 & 1
\end{array}\right)
$$

\subsection{The augmented subshift of finite type}

We can augment the alphabet of $\Sigma$ by an extra symbol, which we denote by 0 [17]. We then associate a subshift of finite type $\tilde{\Sigma}$, where in addition to the transitions allowed by $A$ we also allow transitions from 0 to 0 , and from all the other symbols to 0 , i.e., let

$$
\tilde{A}=\left(\begin{array}{ccccccccc}
1 & 1 & 1 & 1 & \ldots & 1 & 1 & 1 & \ldots \\
0 & 1 & 1 & 1 & \ldots & 0 & 1 & 1 & \ldots \\
0 & 1 & 1 & 1 & \ldots & 1 & 0 & 1 & \ldots \\
0 & 1 & 1 & 1 & \ldots & 1 & 1 & 0 & \ldots \\
\vdots & \vdots & \vdots & \vdots & \vdots & \vdots & \vdots & \vdots & \vdots \\
0 & 0 & 1 & 1 & \ldots & 1 & 1 & 1 & \ldots \\
0 & 1 & 0 & 1 & \ldots & 1 & 1 & 1 & \ldots \\
0 & 1 & 1 & 0 & \ldots & 1 & 1 & 1 & \ldots \\
\vdots & \vdots & \vdots & \vdots & \ldots & \vdots & \vdots & \vdots & \vdots
\end{array}\right)
$$

and

$$
\widetilde{\Sigma}=\left\{\left(i_{n}\right)_{n=0}^{\infty} \in \prod_{n=0}^{\infty}\{0,1,2, \ldots, 2 d\}: \widetilde{A}\left(i_{n}, i_{n+1}\right)=1 \text { for } n \geq 0\right\}
$$


Example 5.2 When $d=2$ the associated matrix takes the form

$$
\tilde{A}=\left(\begin{array}{lllll}
1 & 1 & 1 & 1 & 1 \\
0 & 1 & 0 & 1 & 1 \\
0 & 0 & 1 & 1 & 1 \\
0 & 1 & 1 & 1 & 0 \\
0 & 1 & 1 & 0 & 1
\end{array}\right)
$$

The matrix $A$ for $d=2$ appears as a sub-matrix (by deleting the first row and column of $\tilde{A})$.

This space has a corresponding metric and let $\sigma: \widetilde{\Sigma} \rightarrow \widetilde{\Sigma}$ denote the corresponding shift map.

Let $\Sigma^{*}$ denote the set of finite strings of indices. If $\underline{i}=\left(i_{0}, \ldots, i_{n-1}\right) \in \Sigma^{*}$ then we denote its length by $|\underline{i}|=n$. For a given finite string $\underline{i}=\left(i_{0}, \ldots, i_{n-1}\right) \in \Sigma^{*}$ we can naturally associate a group element $g_{i_{0}} g_{i_{1}} \ldots g_{i_{n-1}} \in \Gamma$. Let us also denote

$$
\sigma\left(i_{0}, \ldots, i_{n-1}\right)=\left(i_{1}, \ldots, i_{n-1}\right)
$$

for finite strings with $n \geq 2$. Finally, for a map $r: \Sigma^{*} \rightarrow \mathbb{R}$ if $\underline{i} \in \Sigma^{*}$ with $|\underline{i}|=n$ we can write

$$
r^{n}(\underline{i})=\sum_{j=0}^{n-1} r\left(\sigma^{j} \underline{i}\right)
$$

We can naturally embed $\Sigma \cup \Sigma^{*}$ into $\widetilde{\Sigma}$ (where a finite string $\left(i_{0}, \ldots, i_{n-1}\right)$ is identified with $\left.\left(i_{0}, \ldots, i_{n-1}, 0,0, \ldots\right) \in \widetilde{\Sigma}\right)$. The following simple lemma relates the symbolic dynamics to the geometry. We are grateful to B. Bowditch for help with the proof.

Lemma 5.3 There exists a Hölder continuous function $r: \Sigma \cup \Sigma^{*} \rightarrow \mathbb{R}$ such that if $\underline{i}=\left(i_{0}, \ldots, i_{n-1}\right) \in \Sigma^{*}(n \geq 2)$ then we can write

$$
r^{n}(\underline{i})=d(\gamma, g \gamma)
$$

where $g=g_{i_{0}} g_{i_{1}} \ldots g_{i_{n-1}}$.

Proof For finite words $g=g_{i_{0}} g_{i_{1}} \ldots g_{i_{n-1}} \in \Gamma$ with $n \geq 1$ we can define

$$
r(\underline{i})=\left(d(\gamma, g \gamma)-d\left(\gamma, g_{i_{0}}^{-1} g \gamma\right)\right)
$$

where $n \geq 2$ and set $r\left(i_{0}\right)=d\left(g_{i_{0}} \gamma, \gamma\right)$. It suffices to show that there exists $0<\theta<1$ and $C>0$ such that for any $g=g_{i_{0}} g_{i_{1}} \ldots g_{i_{n-1}} \in \Gamma$ and $h=h_{i_{0}} h_{i_{1}} \ldots h_{i_{m-1}} \in \Gamma$ satisfying that $g_{i_{j}}=h_{i_{j}}$ for $j=0, \ldots, N-1$ with $1 \leq N \leq \min \{n, m\}$ then

$$
\left|\left(d(\gamma, g \gamma)-d\left(\gamma, g_{i_{0}}^{-1} g \gamma\right)\right)-\left(d(\gamma, h \gamma)-d\left(\gamma, g_{i_{0}}^{-1} h \gamma\right)\right)\right| \leq C \theta^{N} .
$$


In particular, $r$ then extends as a Hölder continuous function to $\Sigma \cup \Sigma^{*}$.

To begin, let $g \gamma$ and $h \gamma$ denote the images of $\gamma$ under the action of $g$ and $h$, respectively. It is also convenient to consider the image $g_{i_{0}} \gamma$ of $\gamma$ under $g_{i_{0}}$. Let $x_{g}$ denote the closest point on $g_{i_{0}} \gamma$ to $g \gamma$. Similarly, we denote by $x_{h}$ the closest point on $g_{i_{0}} \gamma$ to $h \gamma$. It is easy to see that:

(1) $x_{g}$ and $x_{h}$ are exponentially close (i.e., there exists $0<\theta_{1}<1$ such that $\left.d\left(x_{g}, x_{h}\right)=O\left(\theta_{1}^{N}\right)\right)$.

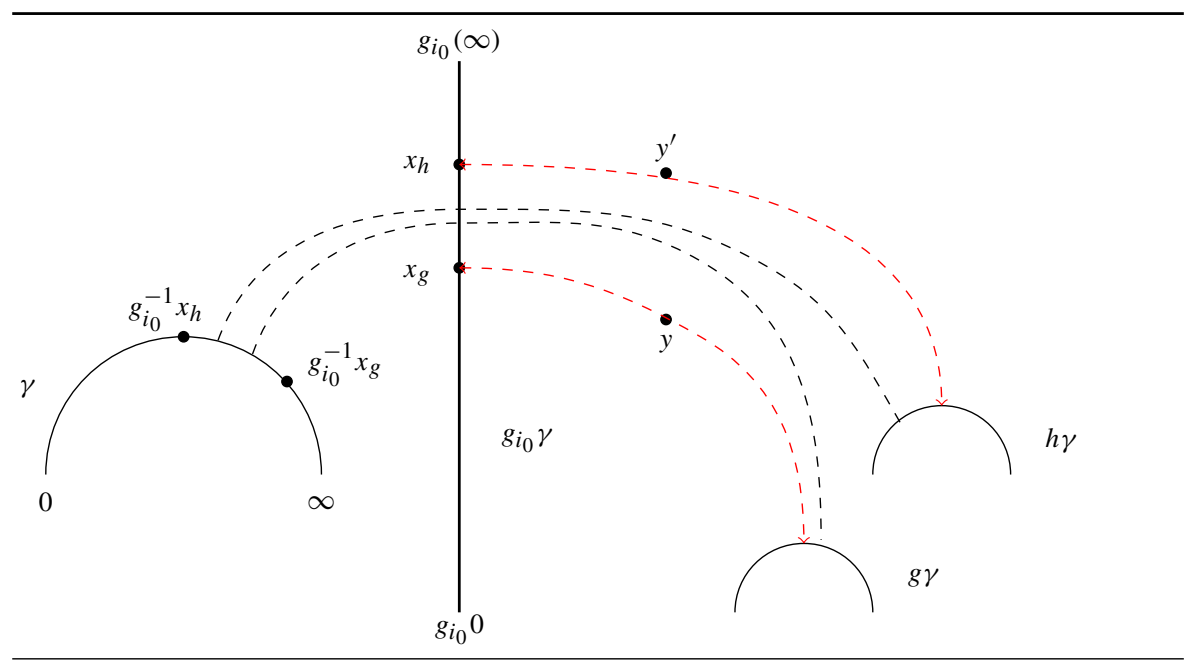

Next, we set $g^{\prime}=g_{i_{1}} g_{i_{2}} \ldots g_{i_{[N / 2]}}\left(=h_{i_{1}} h_{i_{2}} \ldots h_{i_{[N / 2]}}\right) \in \Gamma$. The image $g^{\prime} x_{g}$ lies exponentially close (as a function of $N$ ) to the geodesic from $x_{g}$ to $g \gamma$ and similarly the image $g^{\prime} x_{h}$ lies exponentially close to the geodesic from $x_{h}$ to $h \gamma$. We can then choose points $y$ (on the shortest geodesic arc from $g_{i_{0}} \gamma$ to $g \gamma$ and exponentially close to $g^{\prime} x_{g}$ ) and $y^{\prime}$ (on the shortest geodesic arc from $g_{i_{0}} \gamma$ to $h \gamma$ and exponentially close to $\left.g^{\prime} x_{h}\right)$ such that:

(2) $y$ and $y^{\prime}$ are exponentially close (i.e., there exists $0<\theta_{2}<1$ such that $d\left(y, y^{\prime}\right)=$ $O\left(\theta_{2}^{N}\right)$, say); and

(3) there exists $\epsilon>0$ so that $d\left(x_{g}, y\right), d\left(x_{h}, y^{\prime}\right) \geq \epsilon N$.

Thus, in particular, the geodesic arcs $\left[x_{g}, y\right]$ and $\left[x_{h}, y^{\prime}\right]$ are exponentially close. From (1) we see that the points $g_{i_{0}}^{-1} x_{g}$ and $g_{i_{0}}^{-1} x_{h}$ are both exponentially close to each other, and from (2) and (3) we see that they are exponentially close to the shortest geodesic arcs between $\gamma$ and $g \gamma$, and $\gamma$ and $h \gamma$, respectively. In consequence, there exists $0<\theta<1$ such that:

(a) $\left|d\left(x_{g}, g_{i_{0}}^{-1} x_{g}\right)-d\left(x_{h}, g_{i_{0}}^{-1} x_{h}\right)\right|=O\left(\theta^{n}\right)$;

(b) $\left|\left(d(\gamma, g \gamma)-d\left(g_{i_{0}} \gamma, g \gamma\right)\right)-d\left(x_{g}, g_{i_{0}}^{-1} x_{g}\right)\right|=O\left(\theta^{n}\right)$ or equivalently $\mid(d(\gamma, g \gamma)-$ $\left.d\left(\gamma, g_{i_{0}}^{-1} g \gamma\right)\right)-d\left(x_{g}, g_{i_{0}}^{-1} x_{g}\right) \mid=O\left(\theta^{n}\right) ;$ and 
(c) $\left|\left(d(\gamma, h \gamma)-d\left(g_{i_{0}} \gamma, h \gamma\right)\right)-d\left(x_{h}, g_{i_{0}}^{-1} x_{h}\right)\right|=O\left(\theta^{n}\right)$ or equivalently $\mid(d(\gamma, h \gamma)-$ $\left.d\left(\gamma, g_{i_{0}}^{-1} h \gamma\right)\right)-d\left(x_{h}, g_{i_{0}}^{-1} x_{h}\right) \mid=O\left(\theta^{n}\right)$.

We can then deduce from (a), (b) and (c) that

$$
\begin{aligned}
& \left|\left(d(\gamma, g \gamma)-d\left(\gamma, g_{i_{0}}^{-1} g \gamma\right)\right)-\left(d(\gamma, h \gamma)-d\left(\gamma, g_{i_{0}}^{-1} h \gamma\right)\right)\right| \\
& \quad=\left|(d(\gamma, g \gamma)-d(\gamma, h \gamma))-\left(d\left(\gamma, g_{i_{0}}^{-1} g \gamma\right)-d\left(\gamma, g_{i_{0}}^{-1} h \gamma\right)\right)\right| \\
& \quad \leq\left|d\left(x_{g}, g_{i_{0}}^{-1} x_{g}\right)-d\left(x_{h}, g_{i_{0}}^{-1} x_{h}\right)\right|+\left|d\left(\gamma, g_{i_{0}}^{-1} g \gamma\right)-d\left(\gamma, g_{i_{0}}^{-1} h \gamma\right)\right| \\
& \quad=O\left(\theta^{N}\right)
\end{aligned}
$$

and the result follows.

Remark 5.4 If we consider a more general convex co-compact Kleinian group $\Gamma$ then a similar result holds, where $\Sigma$ is replaced by a more general subshift of finite type. If $\Gamma$ is a co-compact Kleinian group then one can use the associated geodesic flow to obtain a mixing subshift of finite type. However, for convex co-compact Kleinian group $\Gamma$ it is more appropriate to code the strongly Markov groups $\Gamma$. We refer the reader to [21] for more details.

\subsection{Transfer operator}

We can interpret the function $r$ as a function $r: \widetilde{\Sigma} \rightarrow \mathbb{R}$. We can then define a transfer operator $\mathcal{L}_{s}: C^{\alpha}(\tilde{\Sigma}) \rightarrow C^{\alpha}(\tilde{\Sigma})$ by

$$
\mathcal{L}_{s} h(x)=\sum_{\sigma y=x, y \neq 0} e^{-s r(y)} h(y) \text { for } s \in \mathbb{C} .
$$

For $t \in \mathbb{R}$ we can consider the pressure function given by

$$
P(-t)=\sup \left\{h_{\mu}(\sigma)-t \int r d \mu: \mu=T \text {-invariant probability }\right\} \text {. }
$$

We recall that the essential spectral radius of an operator is the spectral radius once we remove isolated eigenvalues of finite multiplicity. We recall the following properties from ([21, Sect. 6]).

Lemma 5.5 The operators $\mathcal{L}_{s}: C^{\alpha}(\Sigma) \rightarrow C^{\alpha}(\Sigma)$ and $\mathcal{L}_{s}: C^{\alpha}(\widetilde{\Sigma}) \rightarrow C^{\alpha}(\widetilde{\Sigma})$ have the same spectra, and both satisfy the following properties.

(1) When $t \in \mathbb{R}$ then $\mathcal{L}_{t}$ has a simple maximal positive eigenvalue $e^{P(t)}$.

(2) The essential spectral radius of $\mathcal{L}_{s}$ is $\frac{e^{P(R e(s))}}{2^{\alpha}}$.

The equality of the two spectra appears as Lemma 2 in [21]. The properties (1), (2) are standard for the transfer operator $\mathcal{L}_{s}: C^{\alpha}(\Sigma) \rightarrow C^{\alpha}(\Sigma)$ (see [19]) and thus extend to $\mathcal{L}_{s}: C^{\alpha}(\widetilde{\Sigma}) \rightarrow C^{\alpha}(\widetilde{\Sigma})$.

Finally, we recall a version of the following well known result of Ruelle [23]. 
Proposition 5.6 The Hausdorf dimension $\delta$ of the limit set $\Lambda$ satisfies $P(\delta)=0$.

Proof By an application of Ruelle's result, the Hausdorff dimension $\delta$ is a zero of the analogous pressure function associated to

(a) the expanding map $T: \Lambda \rightarrow \Lambda$ defined by $T(x)=g_{i}(x)$ if $x \in D_{i}$ and $T(x)=$ $g_{i}^{-1}(x)$ if $x \in D_{i}^{\prime}(i=1, \ldots, d)$ which is modelled by $\sigma: \Sigma \rightarrow \Sigma$, and

(b) the function $\rho: \Sigma \rightarrow \mathbb{R}$ defined by $\rho(x)=\log \left|T^{\prime}(\pi(x))\right|$, where $\pi: \Sigma \rightarrow \Lambda$ is the natural coding map.

It suffices to show that the pressure functions of $\rho: \Sigma \rightarrow \mathbb{R}$ and the restriction $r: \Sigma \rightarrow \mathbb{R}$ agree (since by Lemma 5.5 the pressure function

$$
P(t)=\lim _{n \rightarrow+\infty} \frac{1}{n} \log \left\|\mathcal{L}_{t}^{n}\right\|
$$

is the same using either $r: \widetilde{\Sigma} \rightarrow \mathbb{R}$ or $r: \Sigma \rightarrow \mathbb{R}$ ). We see that for periodic points $x=\sigma^{n} x=\left(x_{n}\right)_{n=0}^{\infty} \in \Sigma$ we have $\rho^{n}(x)=\log \mid\left(T^{n}\right)^{\prime}(\pi(x))=d(g)$ where $d(g)$ corresponds to the displacement along the axis for the element $g \in \Gamma$ associated to the indices $x_{0}, x_{1}, \ldots, x_{n-1}$. However, since $|d(g \gamma, \gamma)-d(g)|$ is uniformly bounded (over $g \in \Gamma$ ) we deduce that $\left|r^{n}(x)-\rho^{n}(x)\right|$ is uniformly bounded (over periodic points $\sigma^{n} x=x$ ). This is sufficient to imply that the pressure functions are the same (and, more particularly, that $\rho, r: \Sigma \rightarrow \mathbb{R}$ are cohomologous [19]).

\section{Proof of Theorem 1.4}

Given any geodesic $\gamma$ with end point outside $F$, we can consider the complex Dirichlet function, formally defined by

$$
\eta(s)=\sum_{g \in \Gamma} e^{-s d(g \gamma, \gamma)} .
$$

The next proposition summarizes the main results we need on $\eta(s)$.

Proposition 6.1 The function $\eta(s)$ satisfies the following properties:

(1) $\eta(s)$ converges provided $\operatorname{Re}(s)>\delta$.

(2) $\eta(s)$ has an analytic extension to a neighbourhood of

$$
\{s \in \mathbb{C}: \operatorname{Re}(s)=\delta\}-\{\delta\} .
$$

(3) $\eta(s)$ has a simple pole at $s=\delta$.

Proof The proof of the proposition is completely analogous to that for Poincare series in [21]. In the case of a free group on 2 generators, say, the main distinction is that we need that the orthogeodesics correspond to reduced words such that additionally satisfy 
(i) $\rho_{1} \rho_{2} \rho_{3} \neq a b a^{-1}$ and $\rho_{1} \rho_{2} \neq b a$; and

(ii) $\rho_{n-2} \rho_{n-1} \rho_{n} \neq b a^{-1} b^{-1}$ and $\rho_{n-1} \rho_{n} \neq b^{-1} a^{-1}$.

For more general free groups there are analogous, if more elaborate conditions arising from finite state automata. (See Proposition 3.4 and Example 3.5 in [13] for more details.)

In particular, we can write

$$
\eta(s)=\sum_{n=1}^{\infty} \mathcal{L}_{s}^{n} 1(\dot{0})
$$

for $\operatorname{Re}(s)>\delta$, where $\dot{0}$ is the sequence of infinitely many 0 s and 1 denotes the constant function.

These results are enough to prove the asymptotic formula. More precisely, we can write

$$
\eta(s)=\int_{1}^{\infty} T^{-s} d N(T)
$$

and then the above proposition allows us to apply the Wiener-Ikehara Tauberian theorem [11].

Theorem 6.2 (Wiener-Ikehara) Let $A(x)$ be a non-negative, monotonic nondecreasing function of $x$, defined for $0 \leq x<+\infty$. Let $d>0$. Suppose that $\int_{1}^{\infty} T^{-s} d A(T)$ converges for $\operatorname{Re}(s)>d$ to the function $f(s)$ and that $f(s)$ is analytic for $\operatorname{Re}(s) \geq d$, except for a simple pole at $s=d$ with residue $C$. Then

$$
A(x) \sim C x^{d} \text { as } x \rightarrow+\infty
$$

The result follows.

\section{Analytic functions and transfer operators}

The transfer operators introduced in Sect. 5 in order to prove the main theorem were, perhaps, a little abstract in nature. In this final section we will consider an alternative, and perhaps more tangible, realization of the connection between the (logarithmic derivative with respect to the second variable of the) Schottky-Klein function and transfer operators, and illustrate its potential usefulness with a simple example.

\subsection{Comparing values at different points}

We recall that the expression (1.2) for the Schottky-Klein function converges whenever $\delta<1$. Several authors have considered the practical problem of explicitly computing its value. 
Given $z, z_{0}, \xi, \xi_{0}$ we can formally write

$$
\frac{w(z, \xi)}{w\left(z, \xi_{0}\right)}=\prod_{g \in \Gamma} \frac{(z-g \xi)}{\left(z-g \xi_{0}\right)}
$$

and since $w(\xi, z)=-w(z, \xi)$ we also have that

$$
\frac{w\left(z, \xi_{0}\right)}{w\left(z_{0}, \xi_{0}\right)}=\frac{w\left(\xi_{0}, z\right)}{w\left(\xi_{0}, z_{0}\right)}=\prod_{g \in \Gamma} \frac{\left(\xi_{0}-g z\right)}{\left(\xi_{0}-g z_{0}\right)} .
$$

If we fix $z_{0}, \xi_{0}$ we can then write

$$
\begin{aligned}
w(z, \xi) & =w\left(z_{0}, \xi_{0}\right)\left(\frac{w\left(z, \xi_{0}\right)}{w\left(z_{0}, \xi_{0}\right)}\right)\left(\frac{w(z, \xi)}{w\left(z, \xi_{0}\right)}\right) \\
& =w\left(z_{0}, \xi_{0}\right)\left(\prod_{g \in \Gamma} \frac{\left(\xi_{0}-g z\right)}{\left(\xi_{0}-g z_{0}\right)}\right)\left(\prod_{g \in \Gamma} \frac{(z-g \xi)}{\left(z-g \xi_{0}\right)}\right) .
\end{aligned}
$$

Taking logarithms gives that

$$
\begin{aligned}
& \log \left(\frac{w(z, \xi)}{w\left(z_{0}, \xi_{0}\right)}\right) \\
& \quad=\sum_{g \in \Gamma} \log (z-g \xi)+\log \left(\xi_{0}-g z\right)-\log \left(z-g \xi_{0}\right)-\log \left(\xi_{0}-g z_{0}\right)
\end{aligned}
$$

We would like to find a more convenient expression to replace the series on the Right Hand Side of (7.2), and for which convergence when $\delta<1$ is more conspicuous. We will again use the transfer operator method, but in the present context it is more appropriate to formulate this in terms of analytic functions, rather than Hölder continuous functions of the preceding sections.

Remark 7.1 In ([7, Chapters 1, 4, 5]) Crowdy also makes use of the expression $\frac{\partial}{\partial \xi} \log \omega(z, \xi)$ in a variety of different settings.

\subsection{Analytic functions and transfer operators}

Given generators (and their inverses) $g_{1}, \ldots, g_{d}, g_{d+1}, \ldots, g_{2 d}$ we can consider domains

$$
U_{i}=\left\{z \in \mathbb{C}:\left|g_{i}^{\prime}(z)\right|>1\right\}
$$

for $i=1, \ldots, 2 d$. These are easily seen to be disjoint, since otherwise one could concatenate the generators corresponding to overlapping domains to contradict the limit set being totally disconnected. We then define $U=\bigsqcup_{i} U_{i}$, which is an open 
neighbourhood of the limit set. Moreover, $U=\mathbb{C}-F$. We can denote by $C^{\omega}(U)$ the Banach space of analytic functions $w: U \rightarrow \mathbb{C}$ which have a continuous extension to the boundary $\partial U$. The associated norm is $\|u\|=\sup _{z \in U}|u(z)|$.

We define a $2 d \times 2 d$ matrix $A$, as before, with entries 0 and 1 .

Definition 7.2 We define a transfer operator $\mathcal{L}: C^{\omega}(U) \rightarrow C^{\omega}(U)$ by

$$
\mathcal{L} w(z)=\sum_{A(j, i)=1} w\left(g_{j} z\right) \text { where } z \in U_{i}
$$

It is easy to see that the constant function is the eigenfunction for the maximal positive eigenvalue $2 d-1$. If $z \in F$ then we define $u_{\xi}(\cdot) \in C^{\omega}(U)$ by $u_{z}(\xi)=$ $\log (z-\xi)$ where $\xi \in D$. Then we can formally write

$$
\log \left(\frac{w(z, \xi)}{w\left(z_{0}, \xi_{0}\right)}\right)=\sum_{n=1}^{\infty}\left(\mathcal{L}^{n} u_{z}(\xi)+\mathcal{L}^{n} u_{\xi_{0}}(z)-\mathcal{L}^{n} u_{z}\left(\xi_{0}\right)-\mathcal{L}^{n} u_{\xi_{0}}\left(z_{0}\right)\right)
$$

for suitable $z_{0}, x, \xi_{0}, \xi$

Moreover, it is possible to see an explicit formula for the derivatives which converges providing $\delta<1$. This gives us an alternative approach to Corollary 2.1. We can write the derivative

$$
\frac{\partial}{\partial \xi} \mathcal{L}^{n} u_{z}(\xi)=\sum_{g_{i_{1}}, \ldots, g_{i_{n}}}\left(g_{i_{1}} \ldots g_{i_{n}}\right)^{\prime}(\xi) w_{z}\left(g_{i_{1}} \ldots g_{i_{n}} \xi\right)
$$

where $w_{z}(\xi)=-\frac{1}{z-\xi}$ and the summation is over generators and inverses such that $g_{i_{j}} \neq g_{i_{j+1}}$. Let us define $\mathcal{M}: C^{\omega}(U) \rightarrow C^{\omega}(U)$ by

$$
\mathcal{M} w(z)=\sum_{A(j, i)=1} g_{j}^{\prime}(\xi) w\left(g_{j} z\right) \quad \text { where } z \in U_{i}
$$

Let us assume that $z, z_{0}, \xi_{0}$ are fixed then we can write the logarithmic derivative as

$$
\begin{aligned}
\frac{\partial}{\partial \xi} \log (w(z, \xi)) & =\frac{\partial}{\partial \xi} \log \left(\frac{w(z, \xi)}{w\left(z_{0}, \xi_{0}\right)}\right) \\
& =\frac{\partial}{\partial \xi}\left(\sum_{n=1}^{\infty} \mathcal{L}^{n} u_{z}(\xi)\right) \\
& =\sum_{n=1}^{\infty} \mathcal{M}^{n} w_{z}(\xi)
\end{aligned}
$$

and formally recover (7.3) from (7.4) by integration. 
Corollary 7.3 If $\delta<1$ then we can write

$$
\log \left(\frac{w(z, \xi)}{w\left(z_{0}, \xi_{0}\right)}\right)=\int_{0}^{1}\left(\sum_{n=1}^{\infty} \mathcal{M}^{n} w_{z}\left(\xi_{0}+t\left(\xi-\xi_{0}\right)\right)\right) d t
$$

It remains to show that (7.4) converges, and that the above analysis holds, provided $\delta<1$ throughout. We will show this in the next subsection.

\subsection{Nuclear operators}

To begin, we recall that in the present context the operator takes a particularly simple form.

Definition 7.4 A bounded linear operator $T: B \rightarrow B$ on a Banach space is called nuclear (with exponentially decaying coefficients) if we can write

$$
T(w)=\sum_{k} \alpha_{k} l_{k}(w) w_{k} \text { for } w \in B
$$

where $w_{k} \in B, l_{k} \in B^{*}$ and $\alpha_{k} \in \mathbb{C}, k \geq 1$, with $\left\|l_{l}\right\|=\left\|w_{k}\right\|=1$ and $\alpha_{k}=O\left(\theta^{k}\right)$.

In particular, nuclear operators are compact and have only isolated eigenvalues of finite multiplicity away from the origin.

We have the following immediate application.

Theorem 7.5 The following can be shown.

(1) The operators $\mathcal{L}: C^{\omega}(U) \rightarrow C^{\omega}(U)$ and $\mathcal{M}: C^{\omega}(U) \rightarrow C^{\omega}(U)$ are both nuclear; and

(2) The operator $\mathcal{M}$ has spectral radius strictly smaller than 1 and the series

$$
\sum_{n=1}^{\infty} \mathcal{M}^{n} u_{z}(\xi)
$$

converges.

Proof The first part is well known cf. [24].

For the second part, we can consider the transformation $T: \Lambda \rightarrow \Lambda$ on the limit set $\Lambda$ given by

$$
T(z)=g_{i}^{-1}(z) \text { if } z \in U_{i}
$$

We can then associate the thermodynamic pressure function $P: \mathbb{R} \rightarrow \mathbb{R}$ defined by

$$
P(t)=\sup \left\{h(m)-t \int \log \left|T^{\prime}\right| d \mu: \mu=\sigma \text {-invariant probability }\right\} .
$$



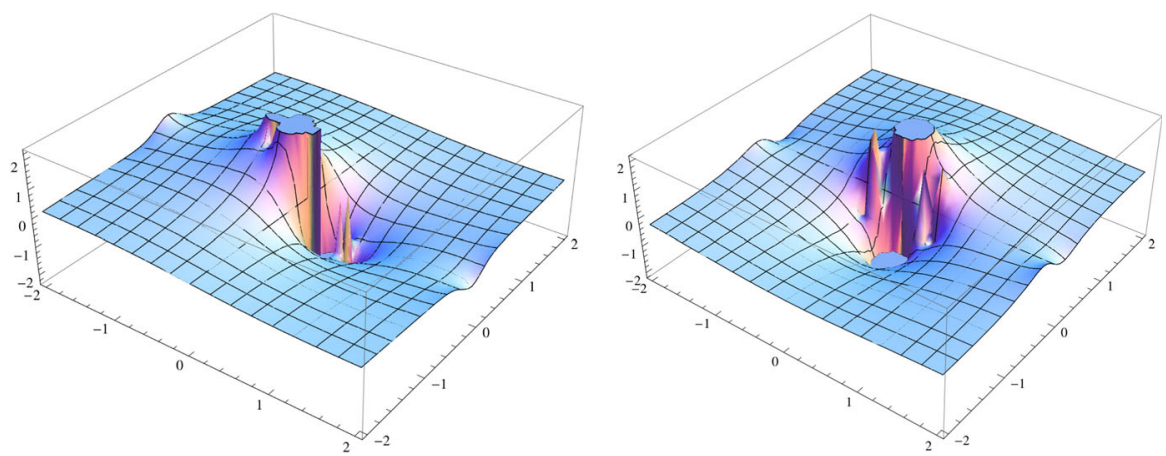

Fig. 4 (i) A plot of $R e\left(\frac{\partial}{\partial \xi} \log w(0, \xi)\right)$; and (ii) a plot of $R e\left(\frac{\partial}{\partial \xi} \log w(0, \xi)\right)$, both in the domain $-2 \leq$ $\operatorname{Re}(\xi), \operatorname{Im}(\xi) \leq 2$

It is easy to see from the definition that $P(t)$ is monotone decreasing, and it is well known that the function is real analytic.

In particular, the spectral radius $\rho(\mathcal{M})$ of this operator will be equal to $P(1)$. It therefore follows from the monotonicity of the function $P(t)$ that if $\delta<1$ then the spectral radius of $\mathcal{M}$ satisfies $\rho(M)=e^{P(1)}<1$, which ensures that the series

$$
\sum_{n=1}^{\infty} \mathcal{M}^{n} u_{z}(\xi)
$$

and related series, converge provided $\delta<1$.

In particular, provided $\delta<1$ we see that the expression on the Right Hand Side of (7.5) is finite by Part (2) of Theorem 7.5. Furthermore, the series converges at an exponential rate given by $\rho(M)=e^{P(1)}<1$.

Example 7.6 (Example 2.2 revisited) We can use (7.4) to estimate the logarithmic derivative (with respect to $\xi$ ) of $w(x, \xi)$. As observed in Chapters 4 and 14 of [7] formula (7.4) can be written in terms of the classical Weierstrass zeta function (Fig. 4). However, as an alternative approach we can use the explicit form of $\mathcal{M}$ and truncate the infinite series in (7.4) to get a reasonable approximation. For example, we fix $z=0$ and plot the real and imaginary parts of $\frac{\partial}{\partial \xi} \log w(0, \xi)$.

Remark 7.7 We can also write the series in Part (2) of Theorem 7.5 in terms of the resolvent, i.e.,

$$
\sum_{n=1}^{\infty} \mathcal{M}^{n}=\frac{1}{1-\mathcal{M}}
$$

and since $\mathcal{M}$ is nuclear we can deduce that (7.5) has a meromophic extension to $\mathbb{C}$. In particular, we can deduce that $w(z, \xi)$ has a meromorphic dependence on the Schottky group $\Gamma$ in the natural sense. 
Remark 7.8 Nuclear operators have the very useful property that they are trace class and lead to very efficient convergence of zeta functions and determinants. However, this corresponds to the fixed points to the actions of elements $g$, rather than the values of orbits of points and thus it is not clear how to take advantage of this property in the present context.

Acknowledgements Funding was provided by H2020 European Research Council (833802).

Open Access This article is licensed under a Creative Commons Attribution 4.0 International License, which permits use, sharing, adaptation, distribution and reproduction in any medium or format, as long as you give appropriate credit to the original author(s) and the source, provide a link to the Creative Commons licence, and indicate if changes were made. The images or other third party material in this article are included in the article's Creative Commons licence, unless indicated otherwise in a credit line to the material. If material is not included in the article's Creative Commons licence and your intended use is not permitted by statutory regulation or exceeds the permitted use, you will need to obtain permission directly from the copyright holder. To view a copy of this licence, visit http://creativecommons.org/licenses/by/4.0/.

\section{References}

1. Baker, H.: Abelian Functions. Cambridge University Press, Cambridge (1897)

2. Bobenko, A.: Introduction to compact Riemann surfaces. In: Bobenko, A., Klein, C. (eds.) Computational Approach to Riemann Surfaces, pp. 3-64. Springer, Berlin (2011)

3. Burnside, W.: On functions determined from their discontinuities and a certain form of boundary condition. Proc. Lond. Math. Soc 22, 346-358 (1891)

4. Crowdy, D.: The Schwarz-Christoffel mapping to bounded multiply connected polygonal domains. Proc. R. Soc. A 461, 2653-2678 (2005)

5. Crowdy, D.: Schwarz-Christoffel mappings to unbounded multiply connected polygonal regions. Math. Proc. Camb. Philos. Soc. 142, 319-339 (2007)

6. Crowdy, D.: Geometric function theory: a modern view of a classical subject. Non-linearity 21, 205219 (2008)

7. Crowdy, D.: Solving Problems in Multiply Connected Domains, NSF-CBMS Regional Conference Series in Applied Mathematics. SIAM, Philadelphia (2020)

8. Crowdy, D., Marshall, J.: Constructing multiply connected quadrature domains. SIAM J. Appl. Math. 64, 1334-1359 (2004)

9. Delillo, T., Kropf, E.: Slit maps and Schwarz-Christoffel maps for multiply connected domains. Electron. Trans. Numer. Anal. 36, 195-223 (2010)

10. Doyle, P.: On the bass note of a Schottky group. Acta Math. 160, 249-284 (1988)

11. Ellison, W., Ellison, F.: Prime Numbers. Wiley, New York (1985)

12. Fenchel, W.: Elementary Geometry in Hyperbolic Space. Walter de Gruyter, Berlin (1989)

13. He, Y.M.: Basmajian-type identities and Hausdorff dimension of limit sets. Ergod. Theory Dyn. Syst. 38, 2224-2244 (2018)

14. Hejhal, D.: Theta Functions, Kernel Functions, and Abelian Integrals, Memoirs of the American Mathematical Society No. 129. American Mathematical Society, Providence (1972)

15. Jenkinson, O., Pollicott, M.: Calculating Hausdorff dimension of Julia sets and Kleinian limit sets. Am. J. Math. 124, 495-545 (2002)

16. Klein, F.: Zur theorie de Abel'schen funcionen. Math. Ann. 36, 1-83 (1890)

17. Lalley, S.: Renewal theorems in symbolic dynamics, with applications to geodesic flow, non-Euclidean tessellations and their fractal limits. Acta Math. 163, 1-55 (1989)

18. Parkkonen, J., Paulin, F.: Counting arcs in negative curvature. In: Aravinda, C.S., Farrell, F.T., Lafont, J.-F. (eds.) Geometry, Topology, and Dynamics in Negative Curvature, LMS Lecture Notes, vol. 426, pp. 289-344 (2016)

19. Parry, W., Pollicott, M.: Zeta functions and the periodic orbit structure of hyperbolic dynamics. Astérisque tome 187-188, 1-268 (1990) 
20. Pollicott, M., Sharp, R.: Orbit counting for some discrete groups acting on simply connected manifolds with negative curvature. Invent. Math. 117, 275-302 (1994)

21. Pollicott, M., Sharp, R.: Comparison theorems and orbit counting in hyperbolic geometry. Trans. Am. Math. Soc. 350, 473-499 (1998)

22. Patterson, S.: A lattice-point problem in hyperbolic space. Mathematika 22, 81-88 (1975)

23. Ruelle, D.: Repellers for real analytic maps. Ergod. Theory Dyn. Syst. 1, 99-107 (1982)

24. Ruelle, D.: Zeta-functions for expanding maps and Anosov Bows. Invent. Math. 34, 231-242 (1976)

25. Schottky, F.: Ueber eine specielle Function, welche bei einer bestimmten linearen Transformation ihres Arguments unverndert bleibt. J. Reine Ang. Math. (Crelle's J.) 10, 227 (1887)

Publisher's Note Springer Nature remains neutral with regard to jurisdictional claims in published maps and institutional affiliations. 